\title{
Comparison of Momelotinib, Pacitinib and Ruxolitinib in patients with Myeloproliferative Neoplasm: An Indirect-Comparison Meta- Analysis of Randomized Controlled Trials
}

Wu Ye

Sichuan University West China Hospital

Xia Wu

Sichuan University West China Hospital

Xue Zheng

Sichuan University West China Hospital

Yuping Gong ( $\nabla$ sichuandaxue@scu.edu.cn )

Sichuan University West China Hospital https://orcid.org/0000-0002-2437-9348

Research article

Keywords: Momelotinib, Pacitinib, Ruxolitinib, Myeloproliferative Neoplasm, Meta-analysis, Indirect comparison

Posted Date: June 2nd, 2020

DOl: https://doi.org/10.21203/rs.3.rs-29345/v1

License: (c) (i) This work is licensed under a Creative Commons Attribution 4.0 International License. Read Full License 


\section{Abstract \\ Background}

The meta-analysis compared the efficacy and safety of momelotinib, ruxolitinib and pacitinib in patients with myeloproliferative neoplasms(MPN), which includes essential thrombocythemia, polycythemia vera and primary myelofibrosis.

\section{Methods}

We searched multiple databases and collected relevant studies before Dec. 20,2019. According to predefined criteria, we included eight qualified phase 3 randomized controlled trials with 2125 patients in the meta-analysis. Statistical analysis used StataMP14 and Indirect Treatment Comparisons software. The meta-analysis is registered with PROSPERO, number CRD42020164271.

\section{Results}

The relative risk ( $95 \%$ confidence interval) of momelotinib vs ruxolitinib, ruxolitinib vs pacritinib and momelotinib vs pacritinib by indirect-comparison are as follows: The $\mathrm{RR}(95 \% \mathrm{Cl})$ for $\geq 35 \%$ reduction in spleen volume from baseline at week $24 / 32$ as determined by magnetic resonance imaging or computed tomography were $0.93(95 \% \mathrm{Cl} 0.69-1.25), 10.87(95 \% \mathrm{Cl} 2.52-46.79)$ and $10.09(95 \% \mathrm{Cl} 2.27-44.74)$; The $\mathrm{RR}(95 \% \mathrm{Cl})$ for $\geq 50 \%$ reduction in total symptom score from baseline at week $24-32$ assessed using the modified Myelofibrosis Symptom Assessment Form were 1.61(95\% Cl0.25-10.15), 4.40(95\% Cl2.13-9.09) and 7.12 (95\% Cl0.91$55.41)$; The RR $(95 \% \mathrm{Cl})$ for the risk of anemia were 0.60(95\% Cl0.20-1.85), 0.80(95\% Cl0.56-1.14) and 0.48 (95\% Cl0.15-1.56); The $\mathrm{RR}(95 \% \mathrm{Cl})$ for thrombocytopenia were $0.69(95 \% \mathrm{Cl} 0.50-0.96), 1.33(95 \% \mathrm{Cl} 0.77-2.30)$ and $0.92(95 \% \mathrm{Cl} 0.48-1.74)$; The RR (95\% $\mathrm{Cl})$ for the risk of fatigue were $1.07(95 \% \mathrm{Cl} 0.71-1.59), 0.82(95 \% \mathrm{Cl} 0.49-1.37)$ and $0.88(95 \% \mathrm{Cl} 0.46-1.68)$; The $\mathrm{RR}(95 \% \mathrm{Cl})$ for the risk of diarrhea were $1.32(95 \% \mathrm{Cl} 0.58-3.01), 0.11(95 \% \mathrm{Cl} 0.03-0.46)$ and $0.39(95 \% \mathrm{Cl} 0.15-1.02)$; The $\mathrm{RR}(95 \% \mathrm{Cl})$ for abdominal pain were $0.95(95 \% \mathrm{Cl} 0.61-1.48), 0.83(95 \% \mathrm{Cl} 0.30-2.25)$ and $0.78(95 \% \mathrm{Cl} 0.26-2.35)$. The RR(95Cl) for the improvement of transfusionindependent of pacritinib vs ruxolitinib was 6.36 (95\% Cl0.79-50.98).

\section{Conclusions}

Excluding the improvement of transfusion-independent and the risk of thrombocytopenia, pacritinib had relatively poorer efficacy and higher risk of adverse events compared with momelotinib or ruxolitinib. Momelotinib had similar or better efficacy and similar or lower risk of adverse events than ruxolitinib while excluding the risk of diarrhea. In general, momelotinib has the best potential therapeutic value in patients with MPN among the three drugs, but further clinical studies are needed to prove it.

\section{Background}

The Philadelphia chromosome-negative chronic myeloproliferative neoplasms (MPN) includes essential thrombocythemia (ET), polycythemia vera $(\mathrm{PV})$ and primary myelofibrosis $(\mathrm{PMF})^{1}$. MF,a rare myeloproliferative neoplasm, is a clonal stem-cell disorder that leads to ineffective erythropoiesis and dysplastic megakaryocytic hyperplasia ${ }^{2}$. Dysregulation with constitutive activation of the Janus kinase-signal transducer and activator of transcription (JAK-STAT) pathway is central to the pathobiology of MF ${ }^{3}$. The JAK family of enzymes includes JAK1, JAK2, JAK3 and TYK2. These molecules attach to the cytosolic domains of cytokine receptors and are essential for cytokine and growth factor signaling. JAK2 is the only member capable of mediating signaling through the 3 myeloid receptors ${ }^{4}$. JAK2 V617F mediates the activation of downstream signaling through STATs (STAT5, STAT3 and STAT1), extra-cellular signal-regulated kinase/mitogen-activated protein kinase, and phosphatidylinositol 3-kinase/AKT/mammalian target of rapamycin pathways, resulting in uncontrolled myeloproliferation ${ }^{5}$. The JAK1/2 inhibitor ruxolitinib improves splenomegaly and symptoms in patients with intermediate-2 or high-risk myelofibrosis, which was approved by the FDA ${ }^{6}$. However, $58-71 \%$ of patients treated with ruxolitinib in clinical trials so far have not achieved the primary endpoint of $35 \%$ or more reduction in spleen volume from baseline assessed by MRI or CT. Furthermore, more than $50 \%$ of patients discontinue ruxolitinib treatment after 3-5 years ${ }^{7}$. When ruxolitinib failure, there are few therapeutic options. Then several small molecular inhibitors were developed 
subsequently, such as momelotinib and pacitinib. Momelotinib is an ATP-competitive small molecule that potently inhibits JAK1/JAK2 kinases (IC(50) $=11$ and $18 \mathrm{nM}$, respectively), with significantly less activity against other kinases, including JAK3 $(\mathrm{IC}(50)=155 \mathrm{nM})^{8}$, which was demonstrated efficacy in patients with primary and secondary myelofibrosis and had potential for efficacious treatment of MPN harboring mutated JAK2 and MPL alleles ${ }^{8,9}$. Pacritinib, a potent JAK2 (IC(50) $=23$ and $19 \mathrm{nM}$ for JAK2(WT) and JAK2(V617F), respectively) and FLT3 (IC(50) = $22 \mathrm{nM})$ inhibitor with selectivity against JAK1 and JAK3 (IC(50) = 1280 and $520 \mathrm{nM}$, respectively ${ }^{10}$, has demonstrated the ability to favorably impact MF-associated splenomegaly and symptom burden ${ }^{11}$. The phase3 clinical trails of momelotinib and pacritinib had been finished. So far, there has been no clinical studies of comparing momelotinib/ruxolitinib with pacritinib by direct head-to-head comparison. The meta-analysis indirectly compared the efficacy and safety of momelotinib, ruxolitinib and pacitinib in patients with MPN.

\section{Methods}

This indirect-comparison meta-analysis was conducted in accordance with the quality of reporting of meta-analysis (PRISMA) statements, and the protocol was registered with PROSPERO, number CRD42020164271.

Inclusive and exclusive criteria

Inclusive criteria: 1 .The experimental group were treated with ruxolitinib, momelotinib or pacritinib; The control group can be blank control, placebo, conventional drugs or one of the three experimental group drugs. 2. There is no ethnic restriction in the study. The patients were diagnosed as myeloproliferative disease and the treatment background of the patients was not limited, which can be the initial treatment or recurrent treatment. 3.The results of the experiments need to include spleen response rate, the improvement of symptom and the risk of adverse events. 4.Studies must be randomized controlled trials (RCTs) and officially published.

Exclusive criteria: 1 .The same study was published repeatedly or the results of different stages of the same clinical trial and only the final or latest results or the results that can be analyzed and utilized shall be taken. 2. The studies had incomplete results and no supplementary data after contacting the author. 3. Basic research and animal experimental research. 4.Studies had a insufficient follow-up time and more than $20 \%$ of the patients lost contacting.

Retrieval methods and studies screening

By searching PubMed, Embase, Medline, Web of Science, Cochrane Library and Chinese Biomedical Database, then we collected all relevant literature before Dec.20,2019. Language is limited to English. The retrieval terms and methods: 1\#:"Primary Myelofibrosis" OR "Essential Thrombocythemia" OR "Polycythemia Vera" OR "Myelofibrosis" OR "Thrombo- cythemia", 2\#:"Ruxolitinib" OR "Momelotinib" OR "Pacritinib" OR"Jakafi" OR "SB1518" OR "CYT387", 3\#: 1\# AND 2\#. Two authors independently reviewed the titles and abstracts to screen potentially eligible studies, then two authors reviewed the full text to screen qualified articles independently. Disagreements between authors were solved by consensus or consultation with a third investigator, if necessary.

Bias risk assessment of the included studies

According to "Cochrane collaboration's tool for assessing risk of bias", the assessment contents include the following aspects: 1.Blind methods; 2.Randomized methods; 3.Allocation concealment; 4.Incomplete outcome data; 5.Selective reporting; 6.0ther biases. Two authors assessed the quality of the studies independently, disagreements between authors were solved by consensus or consultation with a third investigator.

Data extraction

The contents of data extraction were as follows: the first author, year of publication, number of patients, the experimental and control group, follow-up time, $\geq 35 \%$ reduction in spleen volume from baseline as determined by magnetic resonance imaging or computed tomography ${ }^{12}$, achieving a $\geq 50 \%$ reduction in total symptom score (TSS) from baseline assessed using the modified Myelofibrosis Symptom Assessment Form, the risk of hematological and non-hematological adverse events. Two authors extracted the data independently and inputted them into the database.

\section{Statistical analysis}


First step: This meta-analysis used the StataMP14 software to process data from ruxolitinib, momelotinib or pacritinib relevant studies separately. The $\mathrm{p}$-value obtained from chi-squared test and $\mathrm{l}^{2}$ value were used to evaluate the heterogeneity of the study. When $p$-value was bigger than 0.1 and $I^{2}$ was less than $50 \%$, the heterogeneity of the study was considered to be small and the results were analyzed by the fixed-effect-model. When $p$-value was less than 0.1 and l-squared was bigger than $50 \%$, the heterogeneity of the study was considered to be large. When the heterogeneity of the study was large, the meta-analysis would explore the source of heterogeneity or conduct subgroup analysis and all the results were analyzed by the random-effect-model. Relative risk (RR) and 95\% confidence interval (Cl) were used to analyze binary variable data.

Second step: This meta-analysis used the Indirect Treatment Comparisons(ITC) software to compare RR from the first step.

\section{Results}

The screening results of studies

According to the retrieval terms and methods, we obtained 795 studies in the database of PubMed, Embase, Medline, Web of Science, Cochrane Library and Chinese Bio-medical Database. The above-acquired studies were imported into the NoteExpress software for duplicate checking, then the duplicate studies were removed and 345 studies were obtained. After preliminary screening by reading titles and abstracts for potentially eligible studies, there were remaining 47studies. Then we removed 15 nonrandomized controlled trials and read full text for screening eligible studies again. Eventually, eight phase 3 RCTs with 2125 patients were included in quantitative synthesis. Please see flow-process diagram for details in Fig. 1 and the basic characteristics of the included studies in Table 1. 
Table 1

Basic characteristics of the included studies

\begin{tabular}{|c|c|c|c|c|c|c|c|c|}
\hline \multirow[t]{2}{*}{$\begin{array}{l}\text { Included } \\
\text { studies } \\
\text { (phase) }\end{array}$} & \multirow{2}{*}{$\begin{array}{l}\text { Numbers } \\
\text { T C }\end{array}$} & \multirow{2}{*}{$\begin{array}{l}\text { Age } \\
\text { T C }\end{array}$} & \multirow[t]{2}{*}{ MPN } & Male\% & Interventions & \multirow[t]{2}{*}{$\begin{array}{l}\text { Treatment } \\
\text { time(Week) }\end{array}$} & \multirow[t]{2}{*}{$\begin{array}{l}\text { Follow- } \\
\text { up } \\
\text { Time }\end{array}$} & \multirow[t]{2}{*}{ Outcomes } \\
\hline & & & & TC & T C & & & \\
\hline \multirow[t]{2}{*}{$\begin{array}{l}\text { Verstovsek } \\
\text { 2012(III) }\end{array}$} & \multirow[t]{2}{*}{155154} & \multirow[t]{2}{*}{6670} & \multirow[t]{2}{*}{ MF } & \multirow[t]{2}{*}{5157} & $\begin{array}{l}\text { Ruxolitinib } 15 \mathrm{mg} \text { or } \\
20 \mathrm{mg} \text { BID Placebo }\end{array}$ & \multirow[t]{2}{*}{-} & \multirow[t]{2}{*}{$32 W$} & \multirow[t]{2}{*}{$\dagger \ddagger$} \\
\hline & & & & & Maximum 25mg BID & & & \\
\hline \multirow{2}{*}{$\begin{array}{l}\text { Harrison } \\
\text { 2012(III) }\end{array}$} & \multirow[t]{2}{*}{14673} & \multirow[t]{2}{*}{6766} & \multirow[t]{2}{*}{ MF } & \multirow[t]{2}{*}{5758} & $\begin{array}{l}\text { Ruxolitinib } 15 \mathrm{mg} \text { or } \\
20 \mathrm{mg} \text { BID BAT }\end{array}$ & \multirow[t]{2}{*}{-} & \multirow[t]{2}{*}{$48 W$} & \multirow[t]{2}{*}{$\dagger$} \\
\hline & & & & & Maximum 25mg BID & & & \\
\hline \multirow{2}{*}{$\begin{array}{l}\text { Vannucchi } \\
\text { 2015(III) }\end{array}$} & \multirow[t]{2}{*}{110112} & \multirow[t]{2}{*}{6260} & \multirow[t]{2}{*}{ PV } & \multirow[t]{2}{*}{6071} & $\begin{array}{l}\text { Ruxolitinib starting } \\
\text { dose of } 10 \mathrm{mgBID} \text { BAT }\end{array}$ & \multirow[t]{2}{*}{-} & \multirow[t]{2}{*}{$48 W$} & \multirow[t]{2}{*}{$† \ddagger$} \\
\hline & & & & & Maximum 25mg BID & & & \\
\hline Passamonti & 7475 & 6367 & PV & 5363 & $\begin{array}{l}\text { Ruxolitinib starting } \\
\text { dose of } 10 \mathrm{mgBID} \text { BAT }\end{array}$ & 28 & $28 \mathrm{~W}$ & $\ddagger$ \\
\hline & & & & & Maximum 25mg BID & & & \\
\hline Mesa 2017 & 215217 & 6564 & MF & 5855 & $\begin{array}{l}\text { Momelotinib200mgQD } \\
\text { Ruxolitinib 20mg }\end{array}$ & 24 & $24 \mathrm{~W}$ & $† \ddagger$ \\
\hline & & & & & BID & & & \\
\hline Harrison & 10452 & 6669 & MF & 6646 & Momelotinib 200mg & 24 & $24 \mathrm{~W}$ & $† \ddagger$ \\
\hline 2017(III) & & & & & & & & \\
\hline $\begin{array}{l}\text { Mesa } \\
2017(\text { III) }\end{array}$ & 220107 & 6765 & MF & 5756 & $\begin{array}{l}\text { Pacritinib 400mg QD } \\
\text { BAT }\end{array}$ & $\S$ & $23.2 \mathrm{M}$ & $† \ddagger$ \\
\hline $\begin{array}{l}\text { Mascarenhas } \\
\text { 2018(III) }\end{array}$ & 211100 & $\begin{array}{l}69 / 67 \\
69\end{array}$ & MF & $\begin{array}{l}51 / 65 \\
54\end{array}$ & $\begin{array}{l}\text { Pacritinib } \\
400 \mathrm{mgQD} / 200 \mathrm{mgBID} \\
\text { BAT‡ }\end{array}$ & 24 & $24 \mathrm{~W}$ & $\dagger \ddagger$ \\
\hline Notes: & & & & & & & & \\
\hline BATt: Ruxolit & was in 46 & of 52 & ents & tient & h groups were treated & il disease pr & ression. & \\
\hline BAT $\ddagger$ The mos & common B & as ruxolit & nib $\left(45^{\circ}\right.$ & & & & & \\
\hline $\begin{array}{l}\dagger \geq 35 \% \text { reducti } \\
\text { tomography. }\end{array}$ & in spleen & ne at we & $24-3$ & rom ba & as determined by magn & ic resonance & naging or & omputed \\
\hline $\begin{array}{l}\text { łachieving a } \geq \\
\text { Assessment } \mathrm{Fc}\end{array}$ & $\begin{array}{l}50 \% \text { reducti } \\
\mathrm{m} \text {. }\end{array}$ & SS fror & aselir & at week & 2 , assessed using the $m$ & dified Myelofi & osis Sym & tom \\
\hline $\begin{array}{l}\text { Abbreviations: } \\
\text { inhibitors, and }\end{array}$ & $\begin{array}{l}\text { trial; C,cont } \\
\text { ould also }\end{array}$ & AT, best & ailable & herapy,c & sted of any physician-sele & ted treatment & excluding & AK2 \\
\hline $\begin{array}{l}\text { include no trea } \\
\text { symptom scor }\end{array}$ & ent (watch & aiting) & /mp & direc & ment; MF, myelofibros & V, polycyth & vera; & ,total \\
\hline
\end{tabular}

The results of bias risk assessment of the included studies

All studies adopted randomized assignment methods, but only four studies reported the specific randomized assignment methods and assignment concealment, the remaining four studies did not report. The three studies used double-blind methods and the rest were open-label. In the Verstovsek $2012^{13}$, all patients completed evaluation for 24 weeks and the remaining seven studies had incomplete resulting data. Selective reporting and other biases were all unclear. Please see details in Table 2. All studies were phase 
3 large-scale multi-center clinical trails, generally speaking, the risk of bias was relatively small. The Supplementary Appendix was baseline characteristics of the included studies.

Table 2

The quality assessment of the included studies according to Cochrane collaboration's tool for assessing risk of bias

\begin{tabular}{|lllllll|}
\hline Included studies & $\begin{array}{l}\text { Randomized } \\
\text { methods }\end{array}$ & $\begin{array}{l}\text { Blind } \\
\text { methods }\end{array}$ & Allocation concealment & Incomplete outcomes & $\begin{array}{l}\text { Selective } \\
\text { reporting }\end{array}$ & $\begin{array}{l}\text { Other } \\
\text { biases }\end{array}$ \\
\hline Verstovsek 2012 & Unclear & Low risk & Unclear & Low risk & Unclear & Unclear \\
\hline Harrison 2012 & Unclear & Low risk & Unclear & High risk & Unclear & Unclear \\
\hline Vannucchi2015 & Unclear & Low risk & Unclear & High risk & Unclear & Unclear \\
\hline Mesa 2017 & Unclear & Low risk & Unclear & High risk & Unclear & Unclear \\
\hline Harrison 2017 & Low risk & Low risk & Low risk & High risk & Unclear & Unclear \\
\hline Mesa 2017 & Low risk & Low risk & Low risk & High risk & Unclear & Unclear \\
\hline Mascarenhas2018 & Low risk & Low risk & Low risk & High risk & Unclear & Unclear \\
\hline Passamonti2016 & Low risk & Low risk & Low risk & & &
\end{tabular}

Assessments of $\geq 35 \%$ reduction of Spleen Volume and Length as determined by magnetic resonance imaging or computed tomography in patients with MPN

- 1.The Verstovsek(2012), Harrison(2012) ${ }^{14}$ and Vannucchi(2015) ${ }^{15}$ of the ruxolitinib group, the Mesa(2017) $)^{16}$ and Harrison(2017) ${ }^{17}$ of the momelotinib group and the Mesa(2017) ${ }^{18}$ and Mascarenhas(2018) ${ }^{19}$ of the pacritinib group assessed ruxolitinib, momelotinib and pacritinib compared with their corresponding control group, achieving $\geq 35 \%$ reduction in spleen volume from baseline at week 24 or 32 respectively. The pooled $\mathrm{RR}(95 \mathrm{Cl})$ of the ruxolitinib group, the momelotinib group and the pacritinib group versus their corresponding control group were $51.63(95 \% \mathrm{Cl} 14.83-179.71), 0.93(95 \% \mathrm{Cl} 0.69-1.25)$ and 4.75(95\% Cl2.22-10.14) respectively in a fixed-effect-model, as shown in Fig. 2. The control group of the ruxolitinib group and the pacritinib group were placebo or best available therapy (BAT) excluding or just including a small part of JKAi small molecule inhibitors, while the control group of the momelotinib group was ruxolitinib,a dual JAK1/2 inhibitor. The RR greater than 1 favoured the experimental group, otherwise not. As we can see, the ruxolitinib group and the pacritinib group had obviously better effect in spleen response than their corresponding control group, the momelotinib group had similar effect compared with the control group,which was ruxolitinib.

- 2.We used the Indirect Treatment Comparisons (ITC) software to compare Relative Risk from the first step. Having obtained RR of momelotinib vs ruxolitinib, ruxolitinib vs placebo/BAT and pacritinib vs BAT, we can indirectly calculate RR of momelotinib vs pacritinib and ruxolitinib vs pacritinib by ITC software, the RR( $95 \mathrm{Cl})$ of momelotinib vs pacritinib and ruxolitinib vs pacritinib were $10.09(95 \% \mathrm{Cl} 2.27-44.74)$ and $10.87(95 \% \mathrm{Cl} 2.52-46.79)$ respectively. The RR greater than 1 indicated momelotinib or ruxolitinib had better effect than pacritinib, otherwise not. As we can see, momelotinib and ruxolitinib both had better effect in spleen response than pacritinib.

Assessments of $\geq 50 \%$ reduction in total symptom score(TSS) from baseline assessed using the modified Myelofibrosis Symptom Assessment Form

- 1.The Verstovsek(2012), Vannucchi(2015) and Passamonti(2016) ${ }^{20}$ of the ruxolitinib group, the momelotinib group and the pacritinib group compared ruxolitinib, momelotinib and pacritinib with their corresponding control group, achieving a $\geq 50 \%$ reduction in TSS from baseline at week 24-32. The pooled $\mathrm{RR}(95 \mathrm{Cl})$ of ruxolitinib, momelotinib and pacritinib versus their corresponding control group were $7.86(95 \% \mathrm{Cl} 4.86-12.72), 1.61(95 \% \mathrm{Cl} 0.25-10.15)$ and $1.78(95 \% \mathrm{Cl} 1.04-3.07)$ respectively as shown in Fig. 3. The RR greater than 1 favoured the experimental group, otherwise not. The three groups all had better effect in symptomatic improvement compared with their corresponding control group. 
1. 2.The same approach as above-mentioned, we can indirectly calculate RR of momelotinib vs pacritinib and ruxolitinib vs pacritinib by ITC software, the RR( $95 \mathrm{Cl})$ of momelotinib vs pacritinib and ruxolitinib vs pacritinib were $7.12(95 \% \mathrm{Cl} 0.91-55.41)$ and $4.40(95 \% \mathrm{Cl} 2.13-9.09)$ respectively. The RR greater than 1 favoured momelotinib or ruxolitinib, otherwise favoured pacritinib. As we can see, momelotinib and ruxolitinib both had better effect in symptomatic improvement than pacritinib.

All grades of hematologic and non-hematologic adverse events

1.The Verstovsek (2012), Harrison(2012), Vannucchi (2015) and Passamonti(2016) compared ruxolitinib with the control group, the incidence of all grades of hematologic adverse events(anemia and thrombocytopenia) and the incidence of all grades of nonhematologic adverse events (fatigue, diarrhea and abdominal pain) respectively. The pooled RR( $95 \mathrm{Cl}$ ) for anemia, thrombocytopenia, fatigue, diarrhea and abdominal pain of the ruxolitinib group vs the control group were 1.13(95\% Cl1.01-1.27), $1.79(95 \% \mathrm{Cl} 1.15-2.78), 0.87(95 \% \mathrm{Cl} 0.65-1.15), 1.31(95 \% \mathrm{Cl} 0.95-1.79)$ and $0.69(95 \% \mathrm{Cl} 0.28-1.70)$ respectively, as shown in Fig. 4. The RR less than 1 indicated that the experimental group had lower incidence of adverse events compared with the control group, otherwise not. The ruxolitinib group had higher incidence of hematologic adverse events (thrombocytopenia) than the control group and had similar incidence of non-hematologic adverse events compared with the control group. In subgroup analysis, heterogeneity may be due to the type of MPN.

The Mesa(2017) and Harrison(2017) compared momelotinib with ruxolitinib, the incidence of all grades of hematologic adverse events(anemia and thrombocytopenia) and the incidence of all grades of non-hematologic adverse events (fatigue, diarrhea and abdominal pain) respectively. The pooled $\mathrm{RR}(95 \mathrm{Cl})$ for the risk of anemia, thrombocytopenia, fatigue, diarrhea and abdominal pain of the momelotinib group vs the control group were 0.60(95\% Cl0.20-1.85), 0.69(95\% Cl0.50-0.96), 1.07(95\% Cl0.71-1.59), $1.32(95 \% \mathrm{Cl} 0.58-3.01)$ and $0.95(95 \% \mathrm{Cl} 0.61-1.48)$ respectively, as shown in Fig. 5 . The higher the RR value, the higher the incidence of adverse events in the experimental group, otherwise not. As we can see, momelotinib had lower incidence of hematologic adverse events than ruxolitinib and had similar incidence of non-hematologic adverse events compared with ruxolitinib.

The Mesa(2017) and Mascarenhas(2018) compared pacritinib with the control group, the incidence of all grades of hematologic adverse events(anemia and thrombocytopenia) and the incidence of all grades of non-hematologic adverse events (fatigue, diarrhea and abdominal pain) respectively. The pooled $\mathrm{RR}(95 \mathrm{Cl})$ for anemia, thrombocytopenia, fatigue, diarrhea and abdominal pain of the pacritinib group vs the control group were 1.41(95\% Cl1.01-1.99), 1.35(95\% Cl0.97-1.87), 1.05(95\% Cl0.69-1.62), 4.39(95\% Cl3.04-6.35) and 0.83(95\% Cl0.55-1.27) respectively in a fixed-effect model, as shown in Fig. 6 . The RR less than 1 indicated that the experimental group had lower incidence of adverse events compared with the control group, otherwise not. As we can see, the pacritinib group had higher incidence of hematologic adverse events and diarrhea than the control group and had similar incidence of fatigue and abdominal pain compared with the control group.

2.Same approach as above-mentioned, we can indirectly calculate RR of momelotinib vs pacritinib and ruxolitinib vs pacritinib by ITC software. The RR $(95 \mathrm{Cl})$ for the risk of anemia, thrombocytopenia, fatigue, diarrhea and abdominal pain of momelotinib vs pacritinib were $0.48(95 \% \mathrm{Cl} 0.15-1.56), 0.92(95 \% \mathrm{Cl} 0.48-1.74), 0.88(95 \% \mathrm{Cl} 0.46-1.68), 0.39(95 \% \mathrm{Cl} 0.15-1.02)$ and $0.78(95 \% \mathrm{Cl} 0.26-$ 2.35 ) respectively. The $\mathrm{RR}(95 \mathrm{Cl})$ for the risk of anemia, thrombocytopenia, fatigue, diarrhea and abdominal pain of ruxolitinib vs pacritinib were 0.80 (95\%Cl0.56-1.14), 1.33(95\%Cl0.77-2.30), 0.82(95\%Cl0.49-1.37), 0.11(95\%Cl0.03-0.46) and 0.83(95\%Cl0.30-2.25) respectively. The RR less than 1 indicated that momelotinib or ruxolitinib had lower incidence of adverse events compared with pacritinib, otherwise not. According to indirect-comparison results, momelotinib had lower incidence of anemia, diarrhea and abdominal pain than pacritinib and had similar incidence of thrombocytopenia and fatigue compared with pacritinib. Ruxolitinib had similar or lower incidence of adverse events than pacritinib excluding thrombocytopenia.

Assessments of the improvement of transfusion-independent

Assessments of the proportion of patients who were transfusion-dependent at baseline changed their classification to transfusionindependent on study, using the International Working Group for Myelofibrosis Research and Treatment response criteria. In the ruxolitinib vs placebo/BAT studies, only the Verstovsek (2012) reported the specific results of that, the RR for the improvement of transfusion-independent was $0.878(95 \% \mathrm{Cl} 0.509-1.516)$. In the pacritinib vs placebo/BAT studies, we adopted fixed-effect model to calculate RR and the pooled RR was $5.587(95 \% \mathrm{Cl} 0.750-41.617)$, as shown in Fig. 7. We indirectly calculated RR of pacritinib vs 
ruxolitinib by ITC software, the RR was $6.36(95 \% \mathrm{Cl} 0.79-50.98)$. The RR greater than one favoured pacritinib. According to indirectcomparison results, pacritinib had much better effect in the improvement of transfusion-independent than ruxolitinib.

The indirect-comparison results of efficacy and safety between momelotinib, ruxolitinib and pacritinib three groups by Indirect Treatment Comparisons software was summarized in Table 3.

Table 3

The indirect-comparison results of the efficacy and safety between momelotinib, ruxolitinib and pacritinib three groups by Indirect Treatment Comparisons software

\begin{tabular}{|c|c|c|c|}
\hline \multirow[t]{2}{*}{ Outcomes } & Momelotinib vs Ruxolitinib & Ruxolitinib vs Pacritinib & Momelotinib vs Pacritinib \\
\hline & $\mathrm{RR}(95 \mathrm{Cl})$ & $\mathrm{RR}(95 \mathrm{Cl})$ & $\mathrm{RR}(95 \mathrm{Cl})$ \\
\hline Spleen response§ & 0.93(95\%Cl0.69-1.25) & $10.87(95 \% \mathrm{Cl} 2.52-46.79)$ & $10.09(95 \% \mathrm{Cl} 2.27-44.74)$ \\
\hline TSS & $1.61(95 \% \mathrm{Cl} 0.25-10.15)$ & $4.40(95 \% \mathrm{Cl} 2.13-9.09)$ & 7.12(95\%Cl0.91-55.41) \\
\hline Anemiat & $0.60(95 \% \mathrm{Cl} 0.20-1.85)$ & 0.80(95\%Cl0.56-1.14) & $0.48(95 \% \mathrm{Cl} 0.15-1.56)$ \\
\hline Thrombocytopeniat & $0.69(95 \% \mathrm{Cl} 0.50-0.96)$ & 1.33(95\%Cl0.77-2.30) & 0.92(95\%Cl0.48-1.74) \\
\hline Fatiguet & $1.07(95 \% \mathrm{Cl} 0.71-1.59)$ & 0.82(95\%Cl0.49-1.37) & $0.88(95 \% \mathrm{Cl} 0.46-1.68)$ \\
\hline Diarrheat & $1.32(95 \% \mathrm{Cl} 0.58-3.01)$ & $0.11(95 \% \mathrm{Cl} 0.03-0.46)$ & $0.39(95 \% \mathrm{Cl} 0.15-1.02)$ \\
\hline Abdominal paint & 0.95(95\%Cl0.61-1.48) & 0.83(95\%Cl0.30-2.25) & $0.78(95 \% \mathrm{Cl} 0.26-2.35)$ \\
\hline \multicolumn{4}{|l|}{ Notes: } \\
\hline \multicolumn{4}{|c|}{$\begin{array}{l}\text { Spleen response§̧ referred to achieving } \geq 35 \% \text { reduction in spleen volume from baseline at week } 24 / 32 \text { as determined by } \\
\text { magnetic resonance imaging or computed tomography in patients with Myeloproliferative Neoplasm. }\end{array}$} \\
\hline \multicolumn{4}{|c|}{$\begin{array}{l}\text { TSS referred to achieving } a \geq 50 \% \text { reduction in TSS (total symptom score) from baseline at week } 24-32 \text { assessed using the } \\
\text { modified Myelofibrosis Symptom Assessment Form in patients with MPN. }\end{array}$} \\
\hline \multicolumn{4}{|c|}{ †The symbol referred to all grades of adverse events. } \\
\hline Abbreviations: $\mathrm{RR}, \mathrm{Re}$ & isk; Cl,Confidence Interval. & & \\
\hline
\end{tabular}

\section{Discussion}

The meta-analysis indirectly compared the efficacy and safety of momelotinib, ruxolitinib and pacitinib in patients with myeloproliferative neoplasms. As for spleen response, momelotinib and ruxolitinib had similar effect and both were better than pacritinib, but pacritinib also had obviously better effect than other drugs excluding JAKi inhibitors. In the pacritinib group vs the control group studies, the most common BAT of the Mascarenhas(2018) was ruxolitinib (45\%), we regarded the control group of pacritinib as drugs excluding JAKi inhibitors in this meta-analysis, so the results may underestimate pacritinib's efficacy. As for the improvement of symptom in patients, the efficacy of drugs in the treatment of patients with MPN was momelotinib > ruxolitinib > pacritinib > BAT (excluded JAKi inhibitors). Assessments of the proportion of patients who were transfusion-dependent at baseline changed their classification to transfusion independent on study, using the International Working Group for Myelofibrosis Research and Treatment response criteria, pacritinib had better effect than ruxolitinib obviously. As for safety, the risk of all grades of anemia was momelotinib < ruxolitinib $\leqq$ pacritinib; The risk of all grades of thrombocytopenia was momelotinib $\approx$ pacritinib < ruxolitinib; The risk of all grades of fatigue was momelotinib $\approx$ ruxolitinib $\leqq$ pacritinib; The risk of all grades of diarrhea was ruxolitinib < momelotinib < pacritinib; The risk of all grades of abdominal pain was momelotinib $\approx$ ruxolitinib $\leqq$ pacritinib.

\section{Conclusions}

In conclusion, pacritinib compared with momelotinib or ruxolitinib, had relatively poorer efficacy and higher risk of adverse events excluding the risk of thrombocytopenia and the improvement of transfusion-independent in patients with MPN. Momelotinib had similar or better efficacy and similar or lower risk of adverse events than ruxolitinib, excluding the risk of diarrhea. Generally, among the three JAKi inhibitors that were included in the study, momelotinib has the best potential therapeutic value in patients with MPN, 
followed by ruxolitinib and the last one was pacritinib, but pacritinib had a unique advantage in the improvement of transfusionindependent. The meta-analysis was the first study to compare the efficacy and safety of momelotinib, ruxolitinib and pacitinib in patients with MPN by indirect-comparison, so it is likely to provide important information for drug selection in the treatment of MPN in the future. But the meta-analysis also has several limitations, the number of studies that were included were relatively not enough and overall survival rate of patients was not compared, so further clinical studies are needed to prove the results.

\section{Abbreviations}

MPN:myeloproliferative neoplasms, ET:essential thrombocythemia, PV:polycythemia vera, PMF:primary myelofibrosis, MF:myelofibrosis, BAT:best available therapy, RR:relative risk, Cl:confidence interval, ITC:Indirect Treatment Comparisons, RCTs:randomized controlled trials, JAK-STAT:Janus kinase-signal transducer and activator of transcription, TSS:total symptom score, VS:versus

\section{Declarations}

\section{Ethics approval and consent to participate:}

This article does not contain any studies with human participants or animals performed by any of the authors.

\section{Consent for publication:}

Not applicable.

Availability of data and materials:

Not applicable.

Competing interests:

The authors declare that they have no competing interests.

\section{Funding:}

The work was supported by the Foundation of the Science and Technology Department of Sichuan Province (NO.2019YFS0026).

\section{Authors' contributions:}

WY and YPG contributed to the study conception and design. Material preparation, data collection and analysis were performed by WY, XW, XZ and YPG. The first draft of the manuscript was written by WY, and all authors commented on previous versions of the manuscript. All authors read and approved the final manuscript.

\section{Acknowledgments:}

Not applicable.

\section{References}

1. Mikkelsen SU, Kjaer L, Bjørn ME. et al: Safety and efficacy of combination therapy of interferon- $a 2$ and ruxolitinib in polycythemia vera and myelofibrosis. Cancer Med. 2018;7:3571-81.

2. Hanif A, Hari PN, Atallah E. et al: Safety of ruxolitinib therapy prior to allogeneic hematopoietic stem-cell transplantation for myeloproliferative neoplasms. Bone Marrow Transplant. 2016;51:617-8. 
3. Masarova L, Verstovsek S, Hidalgo-Lopez JE. et al: A phase 2 study of ruxolitinib in combination with azacitidine in patients with myelofibrosis. Blood. 2018;132:1664-74.

4. Shammo JM, Stein BL. Mutations in MPNs: prognostic implications, window to biology, and impact on treatment decisions. Hematology. 2016;2016:552-60.

5. Liu F, Zhao X, Perna F. et al: JAK2V617F-mediated phosphorylation of PRMT5 downregulates its methyltransferase activity and promotes myeloproliferation. Cancer Cell. 2011;19:283 - 94.

6. Talpaz M, Erickson-Viitanen S, Hou K. et al: Evaluation of an alternative ruxolitinib dosing regimen in patients with myelofibrosis: an open-label phase 2 study. J Hematol Oncol. 2018;11:101.

7. Harrison CN, Schaap N, Vannucchi AM. et al: Janus kinase-2 inhibitor fedratinib in patients with myelofibrosis previously treated with ruxolitinib (JAKARTA-2): a single-arm, open-label, non-randomised, phase 2, multicentre study. The Lancet Haematology. 2017;4:e317-24.

8. Pardanani A, Lasho T, Smith G. et al: CYT387, a selective JAK1/JAK2 inhibitor: in vitro assessment of kinase selectivity and preclinical studies using cell lines and primary cells from polycythemia vera patients. Leukemia. 2009;23:1441-5.

9. Verstovsek S, Courby S, Griesshammer M. et al: A phase 2 study of momelotinib, a potent JAK1 and JAK2 inhibitor, in patients with polycythemia vera or essential thrombocythemia. Leuk Res. 2017;60:11-7.

10. William AD, Lee AC, Blanchard S. et al: Discovery of the macrocycle 11-(2-pyrrolidin-1-yl-ethoxy)-14,19-dioxa-5,7,26-triazatetracyclo[19.3.1.1(2,6).1(8,12)] heptacosa-1(25),2(26),3,5,8,10,12(27),16,21,23-decaene (SB1518), a potent Janus kinase 2/fms-like tyrosine kinase-3 (JAK2/FLT3) inhibitor for the treatment of myelofibrosis and lymphoma. J Med Chem. 2011;54:4638-58.

11. Diaz AE, Mesa RA. Pacritinib and its use in the treatment of patients with myelofibrosis who have thrombocytopenia. Future Oncol. 2018;14:797-807.

12. Mesa RA, Kiladjian JJ, Verstovsek S. et al: Comparison of placebo and best available therapy for the treatment of myelofibrosis in the phase 3 COMFORT studies. Haematologica. 2014;99:292-8.

13. Verstovsek S, Mesa RA, Gotlib J. et al: A Double-Blind, Placebo-Controlled Trial of Ruxolitinib for Myelofibrosis. N Engl J Med. 2012;366:799-807.

14. Harrison C, Kiladjian J, Al-Ali HK. et al: JAK Inhibition with Ruxolitinib versus Best Available Therapy for Myelofibrosis. N Engl J Med. 2012;366:787-98.

15. Vannucchi AM, Kiladjian JJ, Griesshammer M. et al: Ruxolitinib versus Standard Therapy for the Treatment of Polycythemia Vera. N Engl J Med. 2015;372:426-35.

16. Mesa RA, Kiladjian JJ, Catalano JV. et al: SIMPLIFY-1: A Phase III Randomized Trial of Momelotinib Versus Ruxolitinib in Janus Kinase Inhibitor-Naive Patients With Myelofibrosis. J Clin Oncol. 2017;35:3844-50.

17. Harrison CN, Vannucchi AM, Platzbecker U. et al: Momelotinib versus best available therapy in patients with myelofibrosis previously treated with ruxolitinib (SIMPLIFY 2): a randomised, open-label, phase 3 trial. The Lancet Haematology. 2018;5:e73-81.

18. Mesa RA, Vannucchi AM, Mead A. et al: Pacritinib versus best available therapy for the treatment of myelofibrosis irrespective of baseline cytopenias (PERSIST-1): an international, randomised, phase 3 trial. The Lancet Haematology. 2017;4:e225-36.

19. Mascarenhas J, Hoffman R, Talpaz M. et al: Pacritinib vs Best Available Therapy, Including Ruxolitinib, in Patients With Myelofibrosis. JAMA Oncology. 2018;4:652.

20. Passamonti F, Griesshammer M, Palandri F. et al: Ruxolitinib for the treatment of inadequately controlled polycythaemia vera without splenomegaly (RESPONSE-2): a randomised, open-label, phase 3b study. Lancet Oncol. 2017;18:88-99.

\section{Figures}


Relevant clinical trial studies obtained through database retrieval $(n=795)$

Access to relevant studies through other resources $(n=0)$
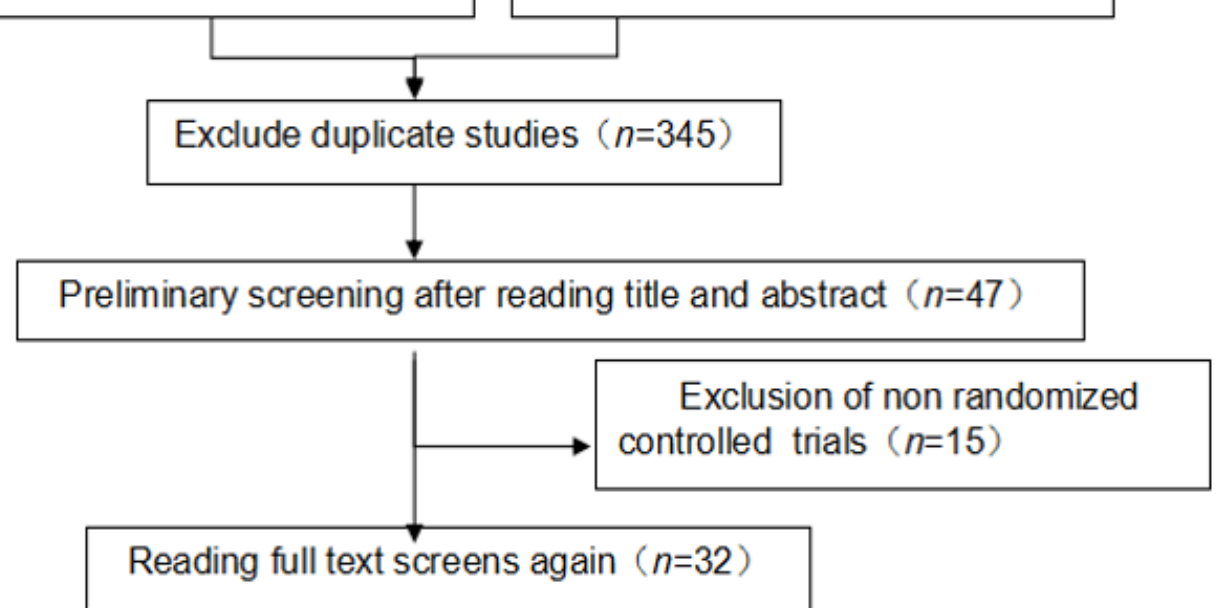

Exclusion $(n=24)$

-No-data-available $(n=17)$

-Long term follow-up of the same trial and analysis of different outcomes $(n=6)$

-Follow-up time less than 24weeks $(n=1)$

Studies included in qualitative analysis $(n=8)$

Studies included in quantitative synthesis (meta-analysis) $(n=8)$

Figure 1

Screening process of randomized controlled trials included in the meta-analysis 
$\%$

RR $(95 \%$ Cl)

Weight

Ruxolitinib vs Control group

Vannucchi (2015)

Verstovsek (2012)

Harrison (2012)

Subtotal (k-squared $=0.0 \%, p=0.951$ )

Momelotinib vs Ruxoltinib

Harrison (2017)

Ruben (2017)

Subtotal (l-squared $=0.0 \%, p=0.721$ )

Pacritinib vs Control group

Mascarenhas (2018)

Mesa (2017)

Subtotal ( 1 -squared $=0.0 \%, p=0.598)$

Overall (l-squared $=92.9 \%, p=0.000)$
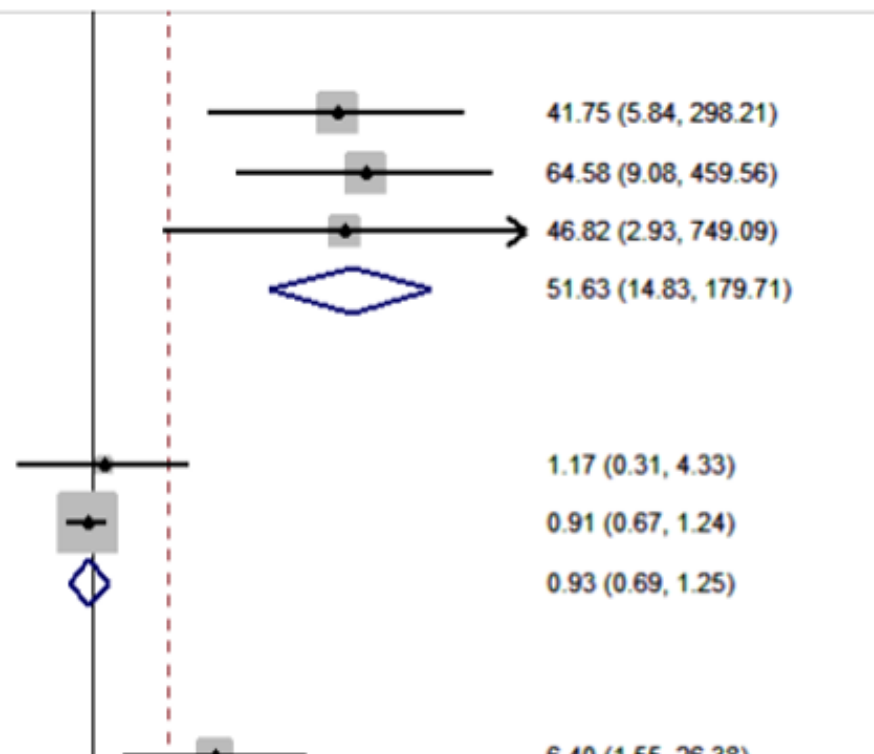

$1.17(0.31,4.33)$

6.00

$0.91(0.67,1.24)$

94.00

$0.93(0.69,1.25)$

100.00
37.26

37.72

25.01

100.00
$6.40(1.55,26.38) \quad 28.74$

$4.09(1.66,10.03) \quad 71.26$

$4.75(2.22,10.14) \quad 100.00$

$3.10(2.42,3.97)$

749

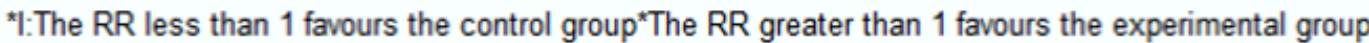

\section{Figure 2}

Forest plots of the pooled Relative Risk (95Confidence Interval) for achieving $\geq 35 \%$ reduction in spleen volume from baseline at week 24/32 as determined by magnetic resonance imaging or computed tomography in patients with Myeloproliferative Neoplasm(MPN) of the ruxolitinib group, the momelotinib group and the pacritinib group versus their corresponding control group respectively. The size of the blocks or diamonds represents the weight for the fixed-effect-model in the meta-analysis 


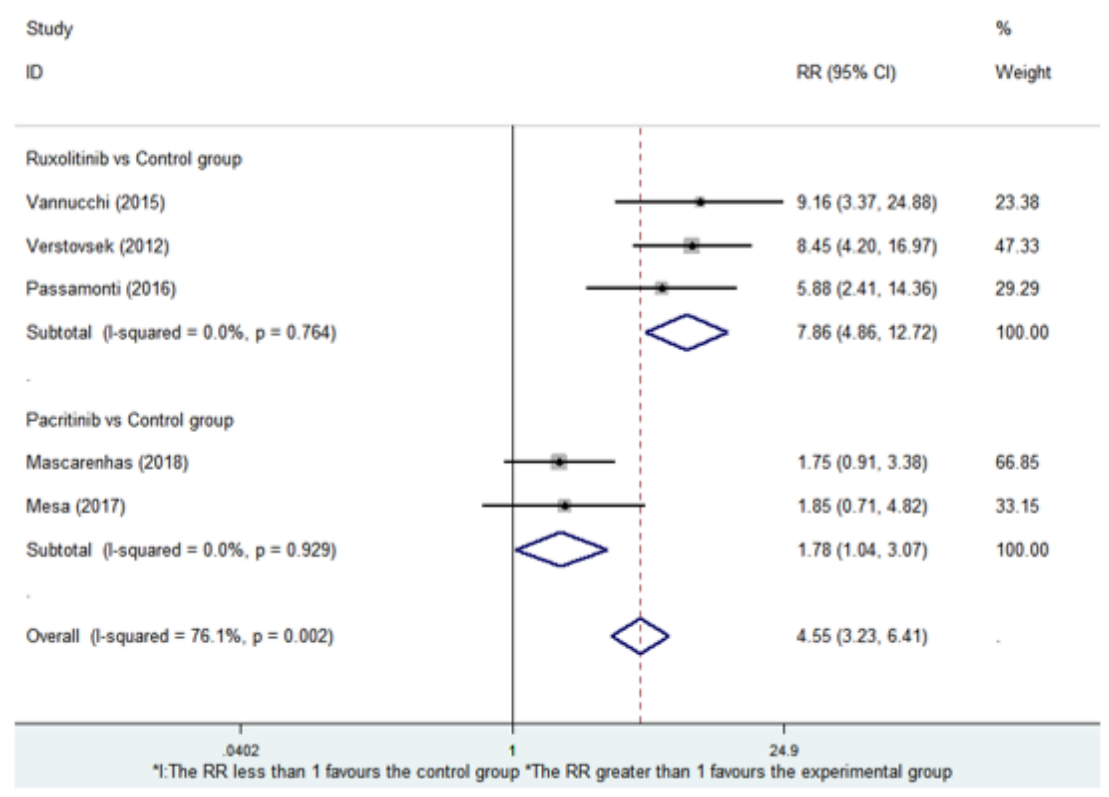

Figure3a: Ruxolitinib and pacritinib vs their corresponding control group

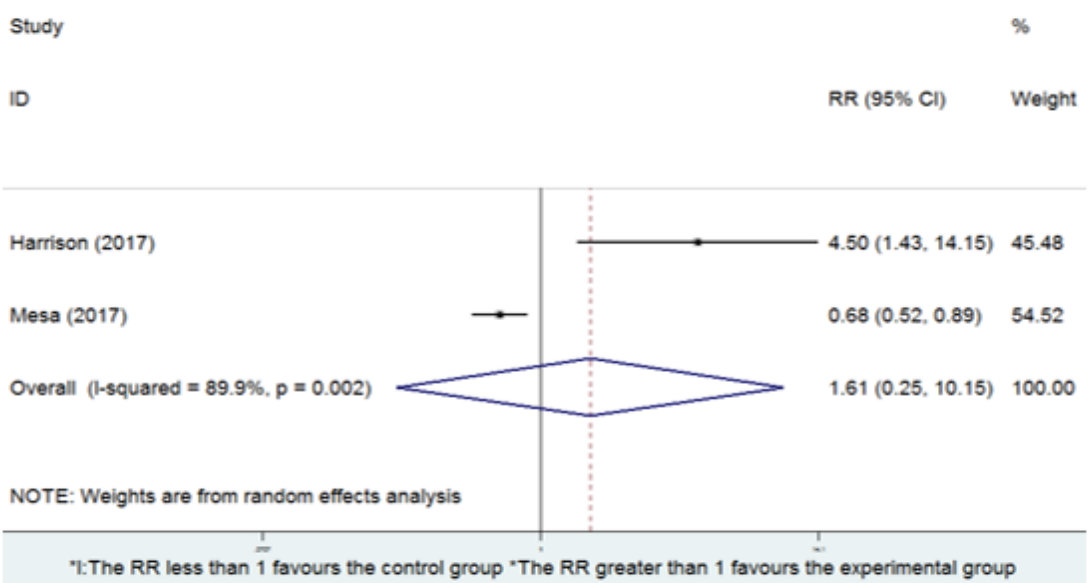

Figure3b: Momelotinib vs control group

\section{Figure 3}

Forest plots of the pooled Relative Risk (95Confidence Interval) for achieving a $\geq 50 \%$ reduction in TSS (total symptom score) from baseline at week 24-32 assessed using the modified Myelofibrosis Symptom Assessment Form in patients with MPN of the ruxolitinib group, the pacritinib group and the momelotinib group versus their corresponding control group respectively. The size of the blocks or diamonds represents the weight for the fixed-effect or random-effect model in the meta-analysis 


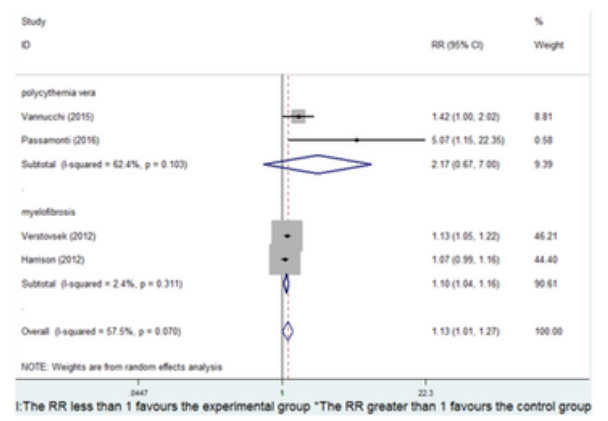

Figureas: The pooled RR(95C) tor anemia of the ruxolitinib group vs the control group

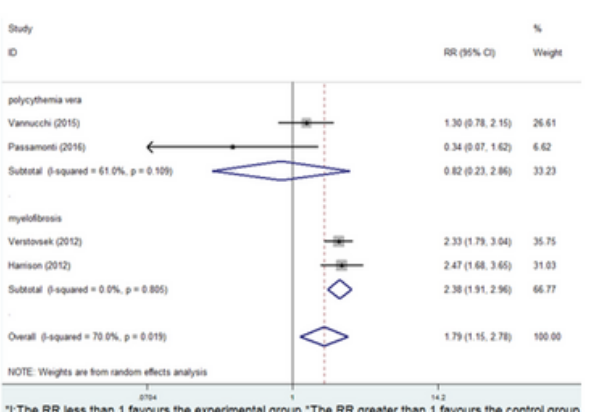

T.The RR less than 1 taveurs the experimential group 'The RR greatior than 1 tavours the control group

Figuresb: The pooled RR(95CI) for thrombocytopenia of the ruxoiltinib group vs the control group
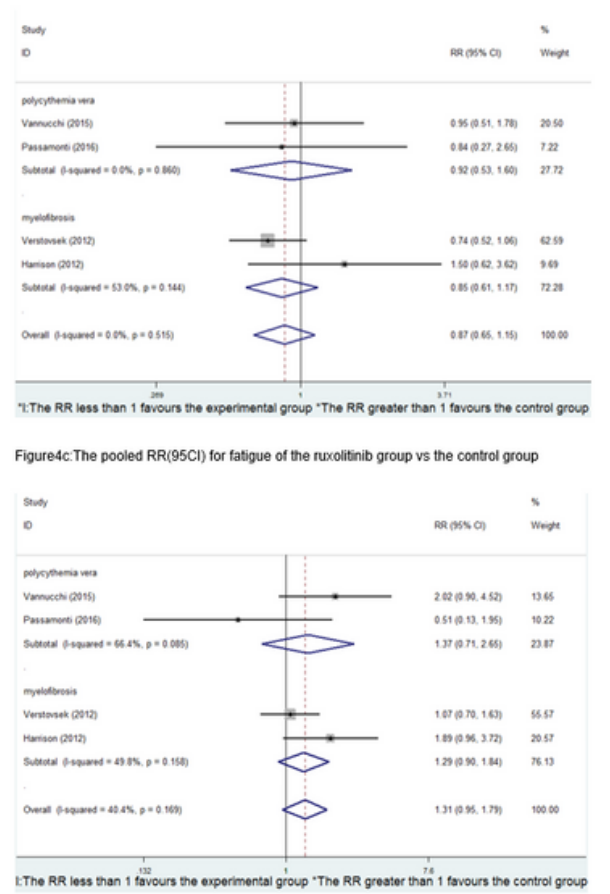

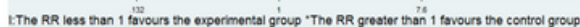

Figure4d: The pooled RR(95CI) for diarriea of the ruxolitiniti group vs the control group

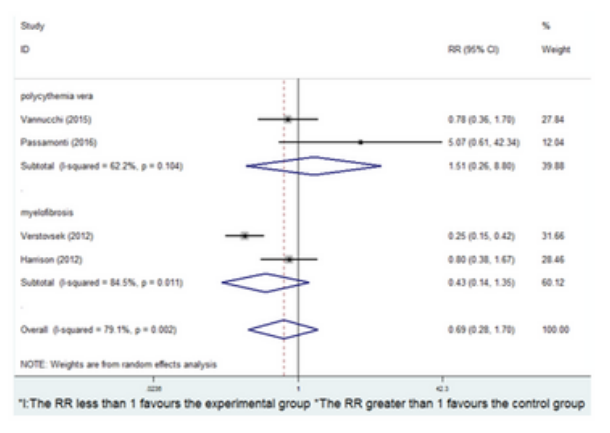

Figure4e: The pooled $R R(95 \mathrm{Cl})$ for abdominal pain of the ruxolatiniti group vs the control group

\section{Figure 4}

Forest plots of the pooled Relative Risk (95Confidence Interval) for all grades of hematologic adverse events(anemia and thrombocytopenia) and non-hematologic adverse events (fatigue, diarrhea and abdominal pain) in patients with MPN of the ruxolitinib group versus the control group respectively. The size of the blocks or diamonds represents the weight for the fixed-effect or random-effect model in the meta-analysis. According to sub-types of MPN(polycythemia vera, myelofibrosis), the meta-analysis conducted subgroup analysis 


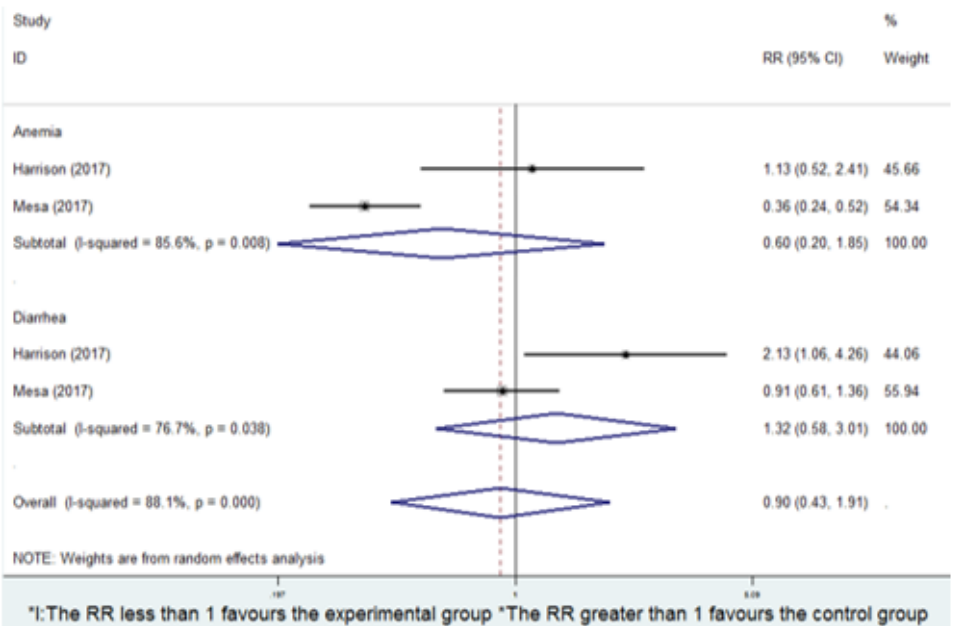

Figure 5a:The pooled RR( $95 \mathrm{Cl})$ for anemia and diarrhea of the momelotinib group vs the control group

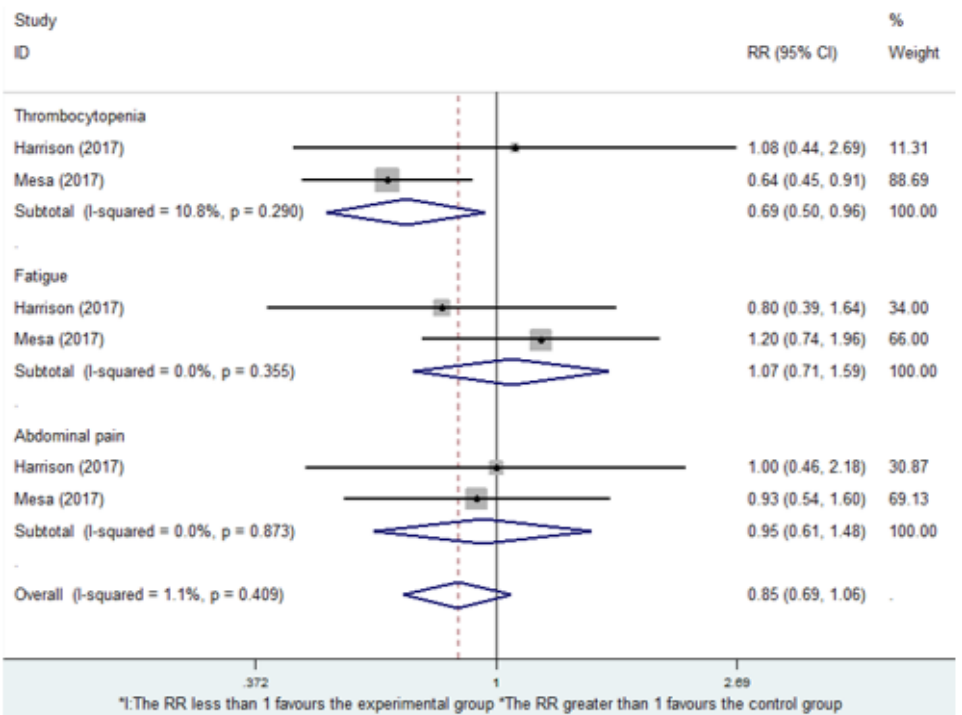

Figure $5 \mathrm{~b}$ :The pooled $\mathrm{RR}(95 \mathrm{Cl})$ for thrombocytopenia, fatigue and abdominal pain of the momelotinib group vs the

control group

\section{Figure 5}

Forest plots of the pooled Relative Risk (95Confidence Interval) for all grades of hematologic adverse events(anemia and thrombocytopenia) and non-hematologic adverse events (fatigue, diarrhea and abdominal pain) in patients with MPN of the momelotinib group versus the control group respectively. The size of the blocks or diamonds represents the weight for the fixedeffect or random-effect model in the meta-analysis 


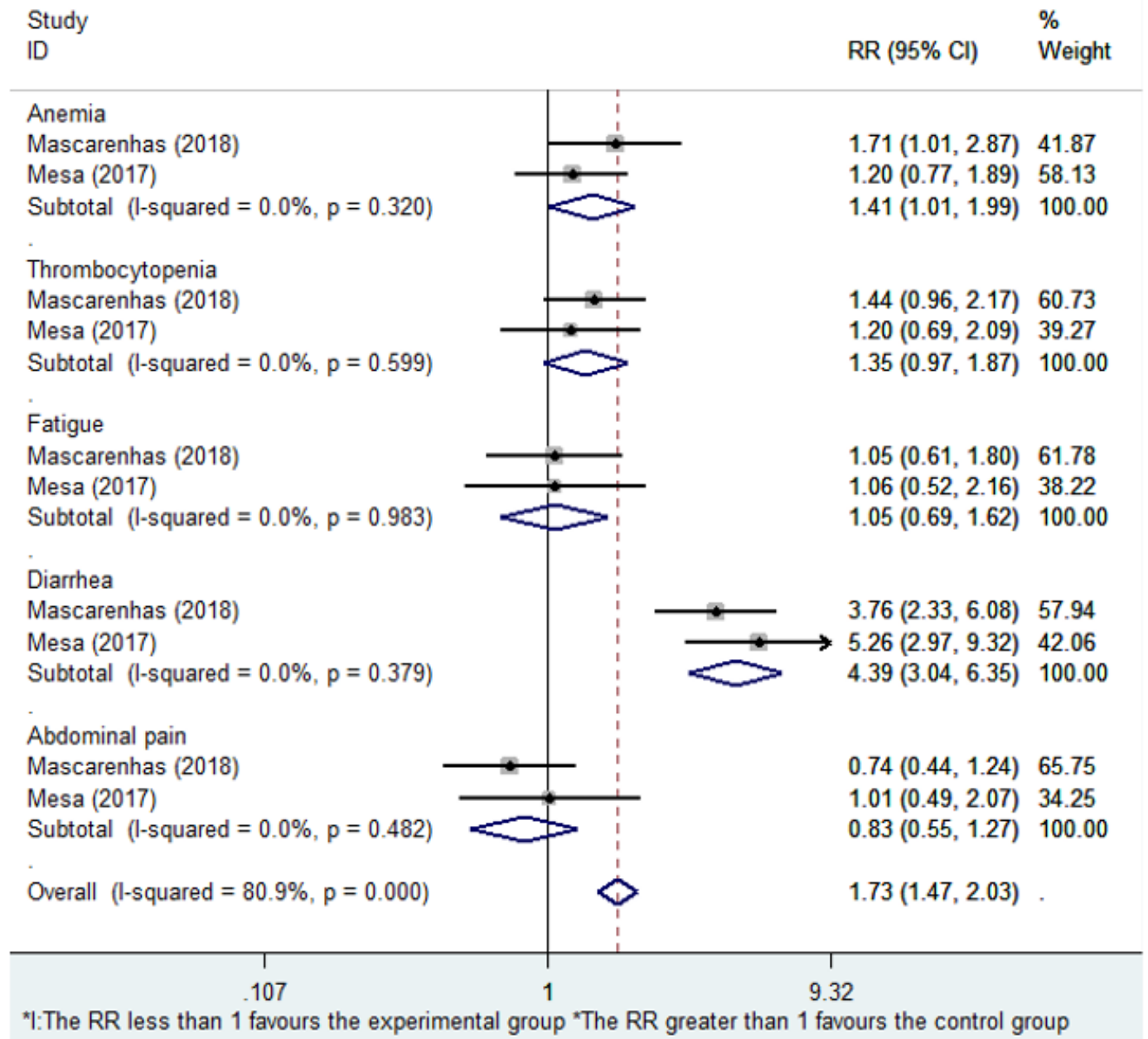

\section{Figure 6}

Forest plots of the pooled Relative Risk (95Confidence Interval) for all grades of hematologic adverse events(anemia and thrombocytopenia) and non-hematologic adverse events (fatigue, diarrhea and abdominal pain) in patients with MPN of the pacritinib group versus the control group respectively. The size of the blocks or diamonds represents the weight for the fixed-effect model in the meta-analysis 
ID

$\operatorname{RR}(95 \% \mathrm{Cl}) \quad$ Weight

Mascarenhas (2018)

Mesa (2017)

Overall $(1-$ squared $=0.0 \%, p=0.526)$

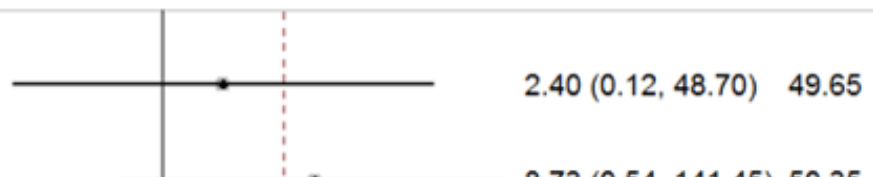

$5.59(0.75,41.62) \quad 100.00$

*I:The RR less than 1 favours the control group *The RR greater than 1 favours the experimental group

\section{Figure 7}

Assessments of the proportion of patients with MPN who were transfusion-dependent at baseline changed their classification to transfusion-independent on study, using International Working Group for Myelofibrosis Research and Treatment response criteria. Forest plots of the pooled Relative Risk (95Confidence Interval) for the improvement of transfusion-independent of the pacritinib group versus the control group.The size of the blocks or diamonds represents the weight for the fixed-effect model in the metaanalysis

\section{Supplementary Files}

This is a list of supplementary files associated with this preprint. Click to download.

- SupplementaryAppendix.pdf

- PRISMAChecklist.doc 